\title{
Effect of atorvastatin on AGEs-induced injury of cerebral cortex via inhibiting NADPH oxidase -NF-KB pathway in ApoE-/- mice
}

zhenhan li ( $\sim$ li_zhenhan@126.com )

Peiye Yang

Shanghai East Hospital

Bo Feng

Shanghai East Hospital

\section{Research}

Keywords: AGEs, Type 2 diabetes, Atorvastatin, Central Nervous System

Posted Date: February 28th, 2020

DOl: https://doi.org/10.21203/rs.3.rs-15490/v1

License: (a) (i) This work is licensed under a Creative Commons Attribution 4.0 International License. Read Full License

Version of Record: A version of this preprint was published at Molecular Biology Reports on November 18th, 2020. See the published version at https://doi.org/10.1007/s11033-020-05998-z. 


\section{Abstract}

\section{Background}

There is close association between type 2 diabetes and cognitive impairment. AGEs-RAGE pathway plays critical role to induce neurodegenerative encephalopathy. Statins can reduce the expression of AGEsinduced RAGE in aorta. It is not clear whether statins have potential benefits on AGEs-induced cognitive impairment. In this study, the effects of atorvastatin on inflammation and oxidation stress in cerebral cortex were investigated and the underlying mechanisms were explored.

\section{Methods}

40 male ApoE-/- mice were randomly divided into four groups: control, AGEs (30mg/ $\mathrm{kg} / \mathrm{day})$, AGEs $(30 \mathrm{mg} / \mathrm{kg} /$ day $)+\operatorname{ALT} 711$ (1 mg/kg/day) and AGEs (30mg/kg/day) + ATV (10mg/kg/day). A $\beta$ formation in cerebral cortex was assessed through Congo red staining and the functional state of neurons were evaluated by Nissl's staining. Immunostaining was performed to assess the accumulation of AGEs in the cerebral cortex. The expressions of mRNA and protein of RAGE, NF-KB p65 and NADPH oxidase p47phox were detected by Real-time PCR and Western blot.

Results

There were significant increases in AGE deposit, A $\beta$ formation and the expressions of RAGE, NF-KB p65 and NADPH oxidase p47phox and decrease in Nissl body in AGEs group compared with control group. ALT711 recovered above change compared with AGEs group. Atorvastatin reduced $A \beta$ formation and suppressed AGEs-induced expressions of NF-KB p65 and NADPH oxidase p47phox. Atorvastatin has little effects on AGE deposit and RAGE expressions.

\section{Conclusions}

Atorvastatin alleviates AGEs-induced neuronal impairment via inhibiting NADPH oxidase-NF-KB pathway and is of benefit in preventing the progression of degenerative encephalopathy.

\section{Background}

Epidemiological study found significant association between type 2 diabetes and moderate degree of cognitive impairment, both for memory and executive functions [1]. It was reported that advanced glycation end products (AGEs) could participate and accelerate the development and progression of diabetic complications, including neurodegenerative diseases [2]. Through direct cytotoxicity and interacting with receptors for AGEs (RAGE), AGEs could intensify the inflammatory response and oxidative stress in brain [3]. AGEs binding to RAGE could transmit the signal from RAGE via redoxsensitive pathways to nuclear factor kappa B (NF-KB)-regulated cytokines [4-7]. AGEs-RAGE system triggers the generation of reactive oxygen species (ROS) by activating nicotinamide adenine dinucleotide phosphate-oxidase (NADPH oxidase) [8], which activates NF-kB and induces subsequent transcription of 
related genes, including endothelin-1, tissue factor, interleukin-1 (IL-1), interleukin-6 (IL-6), and tumor necrosis factor- $a(T N F-a)$ [9-11]. Additionally, activated NF-KB induces the expression of RAGE, which could interact with NF-KB to form a return circuit to amplify the neuronal deterioration process $[9,12]$. Some findings indicated that RAGE-dependent signaling pathway could also facilitate $\beta$ - and $\gamma$-secretase cleavage of amyloid precursor protein to generate $\beta$-amyloid $(A \beta)$ through activating glycogen synthase kinase $3 \beta$ and p38 mitogen-activated protein kinase [13]. Activated cytokines mediated neuronal death, synaptic impairment in chronic inflammatory brain, and ROS in aging could attack vital cell components and ultimately result in cell death.

Apolipoprotein E (ApoE), a main apolipoprotein in brain, transports cholesterol between glial cells and neurons, lack of which leads to neurodegenerative dysfunction. Atorvastatin (ATV), 3-hydroxy-3methylglutaryl coenzyme A reductase inhibitors, exerts cholesterol-lowering activity. Previous studies suggested that statins have properties of anti-inflammatory and immunomodulatory functions and thus exhibits a neuroprotective effect $[14,15]$. Our previous studies demonstrated that atorvastatin could reduce the accumulation of AGEs and the AGEs-induced RAGE expression in aorta independent on glucose levels $(16,17)$. However, whether statins have neuroprotective effects on AGEs-related neurodegenerative encephalopathy is lacking. Based on the pleiotropic effects of statins, we hypothesized that atorvastatin could result in neuroprotective effects via ameliorating neuroinflammation and oxidative stress through down-regulating AGEs-induced activation of RAGE- NADPH oxidase-NF-KB.

\section{Materials And Methods}

\subsection{Animals}

A total of 40 male ApoE knockout $\left(\mathrm{ApoE}^{-/}\right)$mice (age: 6 weeks; weight: $19 \pm 1.7 \mathrm{~g}$ ) were purchased from the Beijing Vital River Laboratory Animal Technology Co.Ltd. (Beijing, China) and were allowed to adjust to their new environment for 2 weeks. The mice were housed at the animal facility of Shanghai East Hospital with free access to food and water and in a pathogen-free environment with a 12-h light/dark cycle. After the adjustment period, the animals were fed with a high-fat diet consisting of $42 \%$ fat, $19 \%$ crude protein and $39 \%$ carbohydrate (Slaccas, Shanghai, China) at a controlled temperature of $22 \pm 2{ }^{\circ} \mathrm{C}$ for 10 weeks. Those mice were randomly divided into four groups: Control $(n=10)$, AGEs $(30 \mathrm{mg} / \mathrm{kg} / \mathrm{day})$ $(n=10)$, AGEs (30 mg/kg/day) + ALT711 (AGEs inhibitors, alagebrium $1 \mathrm{mg} / \mathrm{kg} /$ day) $(\mathrm{n}=10)$ and AGEs $(30 \mathrm{mg} / \mathrm{kg} /$ day $)+$ ATV (atorvastatin $10 \mathrm{mg} / \mathrm{kg} /$ day $)(n=10)$. All animal experimental procedures were in strict accordance with protocols approved by the Ethics Committee of Shanghai East Hospital, Tongji University, and the experiments were performed in accordance with the National Institutes of Health Guidelines for the Use of Laboratory Animals.

\subsection{Preparation Of Ages}


AGEs were prepared as described previously [18]. Briefly, D-glucose, bovine serum albumin (BSA) and penicillin-streptomycin were dissolved in $0.2 \mathrm{mmol} / \mathrm{L} \mathrm{PBS}(\mathrm{pH} 7.4)$ to the final concentrations of $0.5 \mathrm{~mol} / \mathrm{L}, 50 \mathrm{~g} / \mathrm{L}$ and $\mathrm{U} / \mathrm{Lrespectively.} \mathrm{The} \mathrm{reaction} \mathrm{mixture} \mathrm{was} \mathrm{incubated} \mathrm{by} \mathrm{protecting} \mathrm{from} \mathrm{light} \mathrm{for} 24$ hours. They were filtered through $0.22 \mu \mathrm{m}$ filter and then incubated at $37^{\circ} \mathrm{C}$ for 8 weeks. Removing the unconjugated glucose and to dialyze against sterilized PBS for 48 hours. Fluorospectrophotometer with an excitation wave of $370 \mathrm{~nm}$ (the maximum absorption peak was measured at $440 \mathrm{~nm}$ ), and SDS-PAGE (the molecular weight of the material was larger than BSA) that the mixture was glycated-BSA. The glycated-BSA was freeze-dried and stored at $4{ }^{\circ} \mathrm{C}$.

\subsection{Immunohistochemistry Of Ages}

Immunostaining for AGEs was performed as described previously [19]. Briefly, paraffin embedded slide samples prepared as mentioned above were routinely de-waxed, rehydrated and rinsed in $3 \% \mathrm{H}_{2} \mathrm{O}_{2}$ to block endogenous peroxidase. Then the slides were incubated successively with primary antibody against AGEs (1:500, Abcam) and with ABC kit (Beijing Zhongshan Jinqiao Biotechnology, Beijing China). Images were obtained by light microscope. Five sections of cerebral cortex were selected and mean number of positive cells was recorded.

\subsection{Nissl's Staining And Cell Counting}

Nissl's body is used as a marker of the functional state of neurons. Nissl's staining was performed as described previously [20]. Briefly, the tissue sections $(45 \mu \mathrm{m})$ were deparaffinized and subsequently rehydrated using different gradients of ethanol. Then the sections were stained in a $1 \%$ toluidine blue solution for 5-10 minutes, and differentiated in 75\% ethanol for seconds, then rinsed quickly in distilled water. In addition, sections were counterstained with TO-PRO-3 lodide (1:1,000; Life Technologies) for the nonspecific nuclear staining of all cells. At last, sections were sealed with neutral gum. Five sections of cerebral cortex were selected and mean number of positive cells was recorded.

\subsection{Congo Red Staining}

Congo Red histochemical stain may serve as a simple screening tool for investigating if the aggregates in mutant cells have misfolded $\beta$-pleated sheet secondary structures. Congo red staining was performed as described previously [21]. Briefly, brains were perfusion-fixed with $4 \%$ paraformaldehyde and paraffinembedded. For Congo red staining, coronal brain sections were incubated for $5 \mathrm{~min}$ at room temperature in a solution containing $0.2 \%$ Congo red (Biopack, Argentina), $3 \% \mathrm{NaCl}$ and $0.01 \%$ sodium hydroxide in $80 \%$ ethanol. After rinsing, sections were put on gelatin-coated slides, air-dried overnight, dehydrated using ethanol and cleared in xylene. At last, sections were sealed with neutral gum. Sections were visualized using optical microscopy for detection of any orange amyloid plaques. 


\subsection{Quantitative Real-time Polymerase Chain Reaction (qRT- PCR)}

RNA was extracted from cerebral cortex tissue, including RAGE, NF-KB p65 and NADPH oxidase p47phox, using TRIzol reagent (Sigma-Aldrich) according to the standard protocols [22]. Oligonucleotide primers were designed based on Genbank entries for rat RAGE, NF-KB p65, NADPH oxidase p47phox and $\beta$-actin. Primers sequence was presented in Table 1. The following conditions were used for reverse transcription: $25^{\circ} \mathrm{C}$ for $5 \mathrm{~min}, 42^{\circ} \mathrm{C}$ for $60 \mathrm{~min}$, and $70^{\circ} \mathrm{C}$ for $5 \mathrm{~min}$. Each $10 \mu \mathrm{l} \mathrm{PCR}$ contained $2.5 \mu \mathrm{l} \mathrm{cDNA}, 5 \mu \mathrm{l}$ of $2 \times$ SYBR Green PCR Master Mix (Applied Biosystems, Foster city, CA), and 1 pmol of each primer. Quantitative PCR was performed in 96-well optical reaction plates on the AB1 7000 Real-Time PCR System (Applied Biosystems). Relative gene expression was determined by the $\Delta \Delta \mathrm{Ct}$ method, where $\mathrm{Ct}$ meant threshold cycle. All experiments were performed in triplicate.

Table 1

Primers for quantitative PCR.

\begin{tabular}{|lll|}
\hline Gene & Primer & Sequence \\
\hline RAGE & Forward & 5'-GGGAGGCCTGGGAGTAGTAG - 3' \\
\cline { 2 - 3 } & Reverse & 5'-ATTCAGCTCTGCACGTTCCT - 3' \\
NADPH & Forward & 5'-TCCCAAGTGGTTTGACGG-3' \\
& Reverse & 5'-CCTCCTCTTTCTGGCTGTG-3' \\
\multirow{2}{*}{ NF-KB } & Forward & 5'-TCTGCTTCCAGGTGACAGTG - 3' \\
& Reverse & 5'-ATCTTGAGCTCGGCAGTGTT - 3' \\
B-Actin & Forward & 5'-GCCCTGAGGCTCTTTTCCAG - 3' \\
& Reverse & 5'-TGCCACAGGATTCCATACC - 3' \\
\hline
\end{tabular}

\subsection{Western Blotting Analysis}

Western blotting for RAGE, NF-KB p65 and NADPH oxidase p47phox in cerebral cortex was performed as standard protocols [22]. The brain tissue was got out wholly and a part of the cortex was soon separated. Then we homogenized the tissue in RIPA (Beyotime Biotechnology, China) followed with protease inhibitor cocktail (Roche, Basel, Switzerland). The next we utilized the bicinchoninic acid protein assay kit (Beyotime Biotechnology, China) to quantify protein concentration. After that, we separated protein aliquots $(30 \mu \mathrm{g})$ on a $10 \%$ sodium dodecyl sulfate polyacrylamide gel and transferred them onto a polyvinylidene difluoride membrane. The membrane was blocked with $5 \%$ skim milk in TBST and incubated overnight at $4{ }^{\circ} \mathrm{C}$ with one of the following primary antibodies: Rabbit monoclonal RAGE (1:1000, Abcam), NF-KB p65 (1:1000, CST), NADPH oxidase p47phox (1:200, Santa), and $\beta$-actin (1:2000, 
Weiao). Then we washed membranes with TBST for $10 \mathrm{~min} \times 3$ and incubated with horseradish peroxidase-conjugated Goat anti-rabbit secondary antibodies at normal temperature for 1 hour. Using the enhanced chemiluminescence reagent (Millipore, Bedford, MA, USA) and a gel imaging system (Bio-Rad, Hercules, CA, USA) to detect protein bands. The expression of target proteins was semi-qualified as the following formula: Relative coefficient $=$ target protein concentration $/ \beta$-actin concentration .

\subsection{Statistical Analysis}

SPSS 20.0 (IBM, Chicago, IL, USA) was used. Data were expressed as mean \pm standard deviation (SD). One-way analysis of variance was used to compare between four groups. $P<0.05$ was considered as a statistically significant difference.

\section{Results}

\subsection{Deposition of AGEs in cerebral cortex}

As shown in Fig. 1, compared with control group, AGEs-positive cells were significantly increased in cerebral cortex in the AGEs group $(P<0.001)$. While compared with AGEs group, AGEs-positive cells were significantly decreased in the AGEs + ALT711 group $(P<0.01)$, but not in the AGEs + AVT group, suggesting that ALT711 could alleviate AGEs deposit in cerebral cortex but not AVT.

\subsection{The Functional State Of Neurons}

In control group, neurons exhibited a large amount of densely stained toluidine blue granules in the cytoplasm and no histomorphological change were observed (Fig. 2). However, the Nissl bodies dramatically decreased or even disappeared and the histomorphological arrangement were disordered in the AGEs group. Under treatment of atorvastatin or ALT711, there were significantly increase in Nissl bodies and improvement in disordered arrangement compared with AGEs group. These results suggested that AGEs could promote the injury of neurons in $\mathrm{ApoE}^{-/-}$mice, while both AVT and ALT711 could alleviate AGEs-induced injury of neurons.

\subsection{A $\beta$ plaque formation in cerebral cortex}

In order to assess the $A \beta$ plaque formation, Congo red staining was used. The Congo stained amyloid plaque numbers in cortex remarkably increased in the AGEs group compared with control group. While in the atorvastatin group and ALT711 group, Congo red stained plaques was obviously decreased compared with AGEs group (Fig. 3). These results indicated that AVT and ALT711 could efficiently inhibit AGEsinduced $A \beta$ formation in cerebral cortex. 


\subsection{Expression Of RAGE In Cerebral Cortex}

There were significant increases in expression of RAGE mRNA and protein in AGEs group compared with control group. Compared with AGEs group, there were significant decreases in expression of RAGE mRNA and protein in group ALT711, but not in AGEs+ATV group (Fig.4, Fig.5). The results indicated that atorvastatin had little effect on the AGEs-induced expression of RAGE in cerebral cortex.

\subsection{Expression of NADPH oxidase p47phox and NF-kB p65 in cerebral cortex}

There were significant increases in expression of NADPH oxidase p47phox and NF-kB p65 mRNA and protein in AGEs group compared with control group. Compared with AGEs group, there were significant decreases in expression of NADPH oxidase p47phox and NF-KB p65 mRNA and protein in both AGEs+AVT and AGEs+ALT711 groups (Fig.4, Fig.5). These results indicated that AVT could inhibit the transcription and expression of NADPH oxidase p47phox and NF-KB p65 in cerebral cortex.

\section{Discussion}

Diabetic patients are prone to cognitive dysfunction, and the mechanisms include vascular factors and metabolic factors are unclear, among which AGEs plays an important role. The RAGE ligands, including AGEs, high-mobility group box-1 protein, $A \beta$ oligomers and $S 100$ calgranulins, are secreted by microglia and immune cells [23]. Increasing levels of RAGE has been suggested as a cause for amyloid aggregation in the aging and Alzheimer's disease (AD) brains [24]. Excessive accumulation of AGEs and subsequent activation of RAGE are thought to be closely associated with either aging or the development of diabetic complications [25]. RAGE transports circulating $A \beta$ through the blood brain barrier into brain parenchyma and interactions between RAGE and $A \beta$ cause inflammatory responses and oxidative stress, and thus, reduce cerebral blood flow [26]. Glycated $A \beta$ is hypothesized as a more suitable ligand for RAGE, and high levels of AGEs are associated with poorer learning in $A D$ mice. $[25,27]$ A noticeable increase in RAGE levels and oxidative damage is observed in the brain of $A D$ subjects, suggesting a vital role of ROS in the development $A D[28,29]$. Activated microglia play multiple roles in the pathology of $A D$, which contributes to inflammation related neuronal cell death in animal models of $A D[23,30]$. NF-KB plays an important role in transducing inflammatory and pro-apoptotic signals, and RAGE-dependent activation of NF-KB leads to the up-regulation of RAGE itself [31,32]. Additionally, activated microglia secretes large amounts of inflammatory mediators through NF-kB pathways, such as iNOS, TNF-a, and IL-6, which are generally considered prominent factors leading to neuroinflammation and neurotoxicity in neurodegenerative diseases [33]. This study showed that there was obvious deposition of AGEs in the cerebral cortex, which induced neuronal damage with increased expression of RAGE, NADPH oxidase and NF-KB, suggesting that AGEs could activate RAGE-NADPH oxidase-NF-KB pathway to injury the cerebral cortex of mice. 
Neuroprotective mechanisms of statins are a debating topic in the current medical literature. The pleiotropic effects of statins independent of cholesterol-lowering actions have been investigated, including anti-inflammatory, anti-oxidative stress, protection of the neurovascular unit, and facilitating exogenous $A \beta$ degradation [34, 35]. Atorvastatin can down-regulate the RAGE gene directly in aorta [36]. The over-expression of RAGE and NF-KB induced by ischemia after stroke was significantly attenuated by Atorvastatin [14]. But there was no statistically significant difference in decreasing expression of RAGE in our experiment, it suggests atorvastatin might be effective in decreasing RAGE in cerebral cortex slightly. One study found that high-dose atorvastatin could significantly decrease TLR4, TRAF6 and NF-KB, reduce deposited $A \beta$ inducing gliosis and simultaneously ameliorate impairments of spatial learning ability and memory in A $\beta$-injected rat [37]. Statin withdrawal increased NADPH oxidase, an important cellular source of superoxide in the cerebral cortex [38]. In our research, Atorvastatin could be significantly down-regulate NF-KB and NADPH oxidase, leading to decreased nerve injury and deposited of $A \beta$. These results provide evidence for the potent anti-inflammatory and antioxidant activity of atorvastatin. Therefore, atorvastatin could improve cognition function probably related to its effect of decreasing the activation of AGEs-RAGE system, which correlates with block of NADPH oxidase and NF-KB activation. At last, we suppose the proper modulation of RAGE-mediated signaling pathways might be one of the effective therapeutic targets of atorvastatin for AGEs-induced neurodegenerative encephalopathy.

Limitations should be noted in the current study. The concentrations of serum cholesterol esters have not measured, it maybe has a certain effect on the results of our study. $A \beta$ was not assessed through quantitative analysis and which part of the cerebral cortex was not verified. Additionally, though we found that atorvastatin could down-regulated the expression of NF-KB and NADPH oxidase, the first step and the key part were unclear. Moreover, all of these signal pathways were reciprocal causation. Therefore, further researches are still needed to confirm the specific protective mechanism of statins.

\section{Conclusion}

Above all, our study demonstrated that atorvastatin can attenuate AGEs-induced encephalopathy by alleviating inflammation and oxidative stress.

\section{Abbreviations}

AGEs

Advanced glycation end products

RAGE

Receptors for AGEs

NF-KB

Nuclear factor kappa B

ROS

Reactive oxygen species

NADPH oxidase 
Nicotinamide adenine dinucleotide phosphate-oxidase

IL-1

Interleukin-1

IL-6

Interleukin-6

TNF-a

Tumor necrosis factor-a

$A \beta$

$\beta$-amyloid

ApoE

Apolipoprotein E

ATV

Atorvastatin

ALT711

Alagebrium

BSA

Bovine serum albumin

SD

Standard deviation

$A D$

Alzheimer's disease

\section{Declarations}

\section{Acknowledgements}

Not applicable.

\section{Authors' contributions}

Zhenhan Li participated in the design of the study, carried out the immunohistochemistry, performed the statistical analysis and drafted the manuscript. Peiye Yang carried out the animal experiment, RT-PCR and Western blot. Bo Feng conceived of the study, and participated in its design and coordination and helped to draft the manuscript. All authors read and approved the final manuscript.

\section{Funding}

This work was supported by grants from the Key Specialty Construction Project of Pudong Health and Family Planning Commission of Shanghai (PWZzk2017-12).

\section{Availability of data and materials}

The datasets used and analyzed during the current study are included in this published article. 


\section{Ethics approval and consent to participate}

This study was conducted in accordance with the "Guide for the Care and Use of Laboratory Animals" prepared by the National Academy of Sciences and published by the National Institutes of Health and with the approval by the Ethical Committee on Animal Research at Shanghai East Hospital of Tongji University (Shanghai, China)

\section{Consent for publication}

Not applicable.

\section{Competing interests}

The authors declare that they have no competing interests.

\section{References}

1. Bello-Chavolla OY., et al., Pathophysiological Mechanisms Linking Type 2 Diabetes and Dementia: Review of Evidence from Clinical, Translational and Epidemiological Research. Curr Diabetes Rev.2019;15(6):456-470.

2. Byun, K., et al., Advanced glycation end-products produced systemically and by macrophages: A common contributor to inflammation and degenerative diseases. Pharmacol Ther, 2017. 177: p. 4455 .

3. Shen, C., et al., RAGE-Specific Inhibitor FPS-ZM1 Attenuates AGEs-Induced Neuroinflammation and Oxidative Stress in Rat Primary Microglia. Neurochem Res, 2017. 42(10): p. 2902-2911.

4. Yeh, C.H., et al., Requirement for p38 and p44/p42 mitogen-activated protein kinases in RAGEmediated nuclear factor-kappaB transcriptional activation and cytokine secretion. Diabetes, 2001. 50(6): p. 1495-504.

5. Singh, R., et al., Advanced glycation end-products: a review. Diabetologia, 2001. 44(2): p. 129-46.

6. Fan, Q., et al., Candesartan reduced advanced glycation end-products accumulation and diminished nitro-oxidative stress in type 2 diabetic KK/Ta mice. Nephrol Dial Transplant, 2004. 19(12): p. 301220.

7. Yamagishi, S., et al., Role of advanced glycation end products (AGEs) and oxidative stress in diabetic retinopathy. Curr Pharm Des, 2008. 14(10): p. 962-8.

8. Srikanth, V., et al., Advanced glycation endproducts and their receptor RAGE in Alzheimer's disease. Neurobiology of Aging, 2011. 32(5): p. 763-777.

9. Bierhaus, A., et al., Understanding RAGE, the receptor for advanced glycation end products. J Mol Med (Berl), 2005. 83(11): p. 876-86.

10. Bierhaus, A. and P.P. Nawroth, Multiple levels of regulation determine the role of the receptor for AGE (RAGE) as common soil in inflammation, immune responses and diabetes mellitus and its 
complications. Diabetologia, 2009. 52(11): p. 2251-63.

11. Bierhaus, A., et al., Diabetes-associated sustained activation of the transcription factor nuclear factor-kappaB. Diabetes, 2001. 50(12): p. 2792-808.

12. Mattson, M.P. and S. Camandola, NF-kappaB in neuronal plasticity and neurodegenerative disorders. J Clin Invest, 2001. 107(3): p. 247-54.

13. Fang, F., et al., RAGE mediates Abeta accumulation in a mouse model of Alzheimer's disease via modulation of beta- and gamma-secretase activity. Hum Mol Genet, 2018. 27(6): p. 1002-1014.

14. Wang, L., et al., Atorvastatin protects rat brains against permanent focal ischemia and downregulates HMGB1, HMGB1 receptors (RAGE and TLR4), NF-kappaB expression. Neurosci Lett, 2010. 471(3): p. 152-6.

15. Xu, X., et al., Anti-inflammatory and immunomodulatory mechanisms of atorvastatin in a murine model of traumatic brain injury. J Neuroinflammation, 2017. 14(1): p. 167.

16. Feng $\mathrm{B}, \mathrm{Xu} \mathrm{L}$, Wang $\mathrm{H}$, et al. Atorvastatin exerts its anti-atherosclerotic effects by targeting the receptor for advanced glycation end products. BBA - Molecular Basis of Disease, 2011, 1812(9): $1130-1137$.

17. Xu L, Zang P, Feng B, Qian Q. Atorvastatin inhibits the expression of RAGE induced by advanced glycation end products on aortas in healthy Sprague-Dawley rats. Diabetol Metab Syndr, 2014, 6(1):102.

18. Chen, F., et al., RAGE-NF-kappaB-PPARgamma Signaling is Involved in AGEs-Induced Upregulation of Amyloid-beta Influx Transport in an In Vitro BBB Model. Neurotox Res, 2018. 33(2): p. 284-299.

19. Xu L, Xiong Y., et al., Pioglitazone attenuates advanced glycation end products-induced apoptosis and calcification by modulating autophagy in tendon-derived stem cells. J Cell Mol Med. 2020 Feb;24(3):2240-2251

20. Chu, H., Y. Tang, and Q. Dong, Protection of Vascular Endothelial Growth Factor to Brain Edema Following Intracerebral Hemorrhage and Its Involved Mechanisms: Effect of Aquaporin-4. PLoS One, 2013. 8(6): p. e66051.

21. Du, L.Y., et al., Alzheimer's Disease-Related Protein Expression in the Retina of Octodon degus. PLoS One, 2015. 10(8): p. e0135499.

22. Zhang, K.L., D.D. Lou, and Z.Z. Guan, Activation of the AGE/RAGE system in the brains of rats and in SH-SY5Y cells exposed to high level of fluoride might connect to oxidative stress. Neurotoxicol Teratol, 2015. 48: p. 49-55.

23. Chuah, Y.K., et al., Receptor for advanced glycation end products and its involvement in inflammatory diseases. Int J Inflam, 2013. 2013: p. 403460.

24. Chaney, M.O., et al., RAGE and amyloid beta interactions: atomic force microscopy and molecular modeling. Biochim Biophys Acta, 2005. 1741(1-2): p. 199-205.

25. Lubitz, I., et al., High dietary advanced glycation end products are associated with poorer spatial learning and accelerated Abeta deposition in an Alzheimer mouse model. Aging Cell, 2016. 15(2): p. 
309-16.

26. Deane, R.J., Is RAGE still a therapeutic target for Alzheimer's disease? Future Med Chem, 2012. 4(7): p. $915-25$.

27. Li, X.H., et al., Glycation exacerbates the neuronal toxicity of beta-amyloid. Cell Death Dis, 2013. 4: p. e673.

28. Christen, Y., Oxidative stress and Alzheimer disease. Am J Clin Nutr, 2000. 71(2): p. 621s-629s.

29. Huang, W.J., X. Zhang, and W.W. Chen, Role of oxidative stress in Alzheimer's disease. Biomed Rep, 2016. 4(5): p. 519-522.

30. Liu, C., et al., Neuroinflammation in Alzheimer's disease: chemokines produced by astrocytes and chemokine receptors. Int J Clin Exp Pathol, 2014. 7(12): p. 8342-55.

31. Ramasamy, R., et al., Advanced glycation end products and RAGE: a common thread in aging, diabetes, neurodegeneration, and inflammation. Glycobiology, 2005. 15(7): p. 16r-28r.

32. Li, J. and A.M. Schmidt, Characterization and functional analysis of the promoter of RAGE, the receptor for advanced glycation end products. J Biol Chem, 1997. 272(26): p. 16498-506.

33. Xu, S., et al., Inhibition of cathepsin L alleviates the microglia-mediated neuroinflammatory responses through caspase-8 and NF-kappaB pathways. Neurobiol Aging, 2018. 62: p. 159-167.

34. Yamamoto, N., et al., Simvastatin and atorvastatin facilitates amyloid beta-protein degradation in extracellular spaces by increasing neprilysin secretion from astrocytes through activation of MAPK/Erk1/2 pathways. Glia, 2016. 64(6): p. 952-62.

35. Martins, W.C., et al., Atorvastatin Prevents Cognitive Deficits Induced by Intracerebroventricular Amyloid-beta1-40 Administration in Mice: Involvement of Glutamatergic and Antioxidant Systems. Neurotox Res, 2015. 28(1): p. 32-42.

36. Feng, B., et al., Atorvastatin exerts its anti-atherosclerotic effects by targeting the receptor for advanced glycation end products. Biochim Biophys Acta, 2011. 1812(9): p. 1130-7.

37. Wang, S., et al., Atorvastatin Attenuates Cognitive Deficits and Neuroinflammation Induced by Abeta1-42 Involving Modulation of TLR4/TRAF6/NF-kappaB Pathway. 2018. 64(3): p. 363-373.

38. de Oliveira, C.V., et al., Atorvastatin withdrawal elicits oxidative/nitrosative damage in the rat cerebral cortex. Pharmacol Res, 2013. 71: p. 1-8.

\section{Figures}




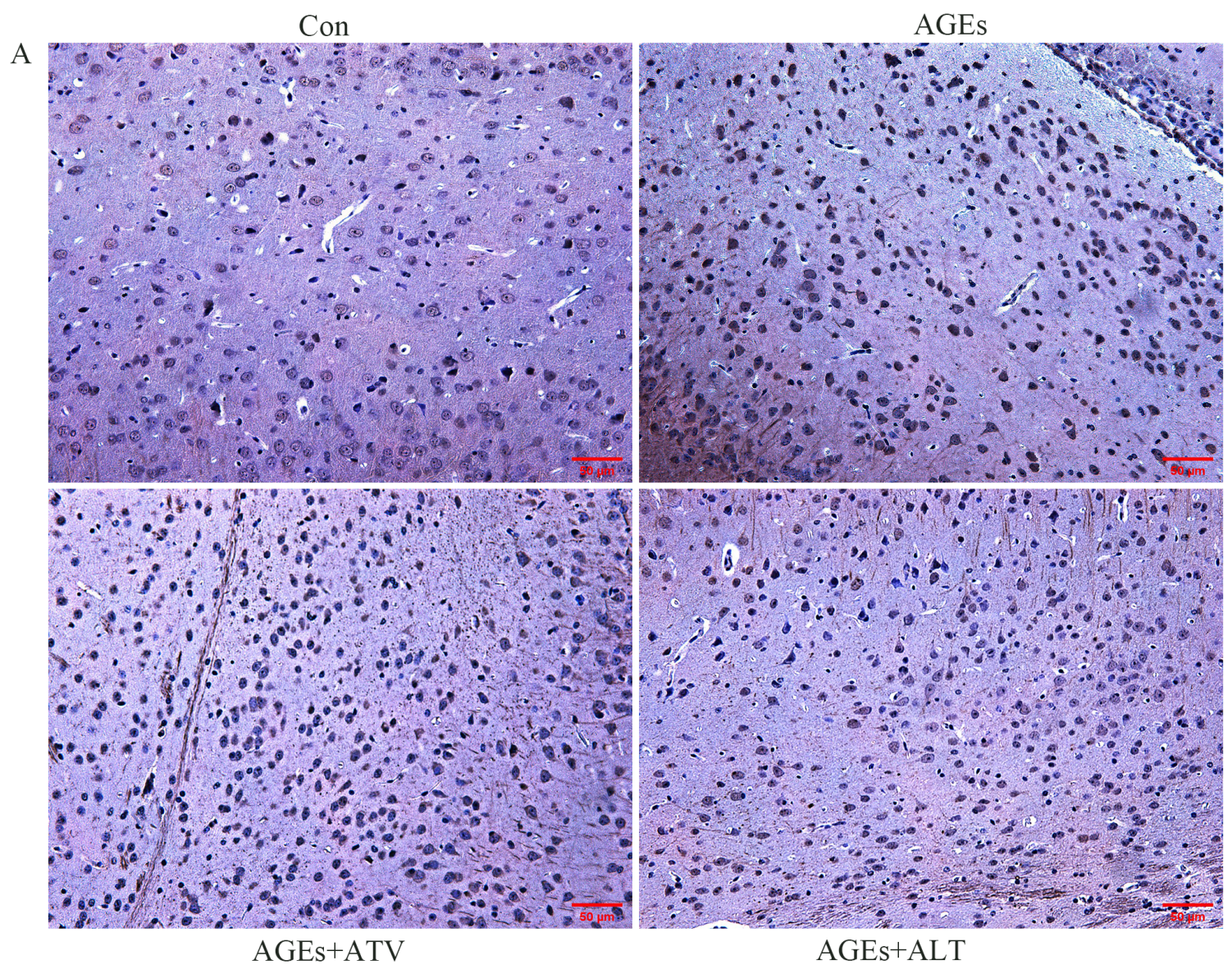

B

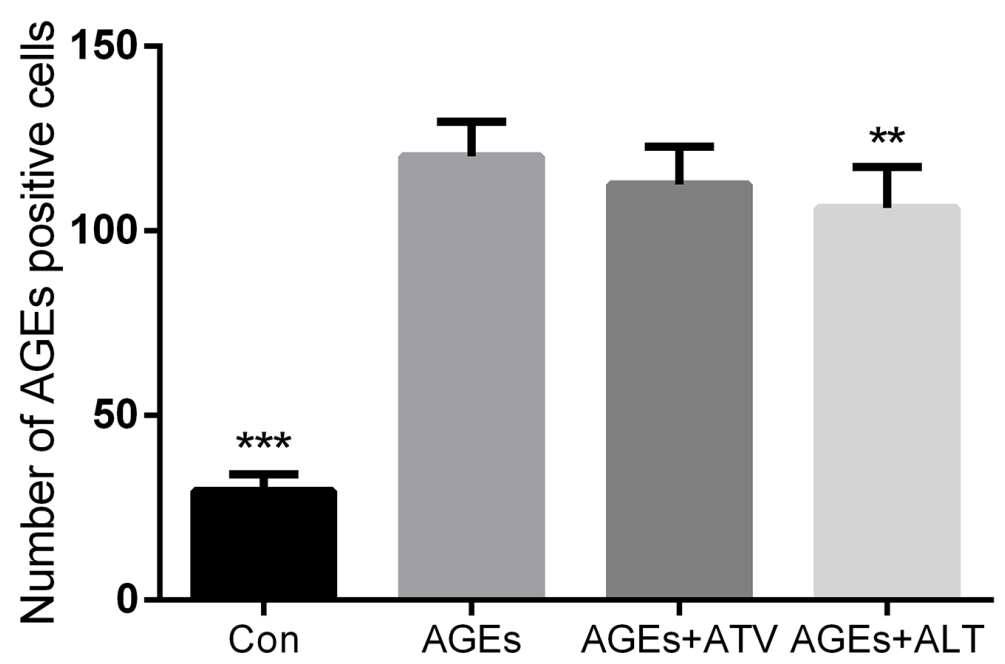

Figure 1

Deposition of AGEs in cerebral cortex. Brain sections with AGEs immunostaining $(A, \times 200$ magnification). Number of AGEs positive cells in cerebral cortex(B). GraphPad values are presented as mean $\pm \mathrm{SEM} . * \mathrm{P}<0.05$ versus the AGEs group, $*^{*} \mathrm{P}<0.01$ versus the AGEs group and $*^{* *} \mathrm{P}<0.001$ versus the AGEs group. 


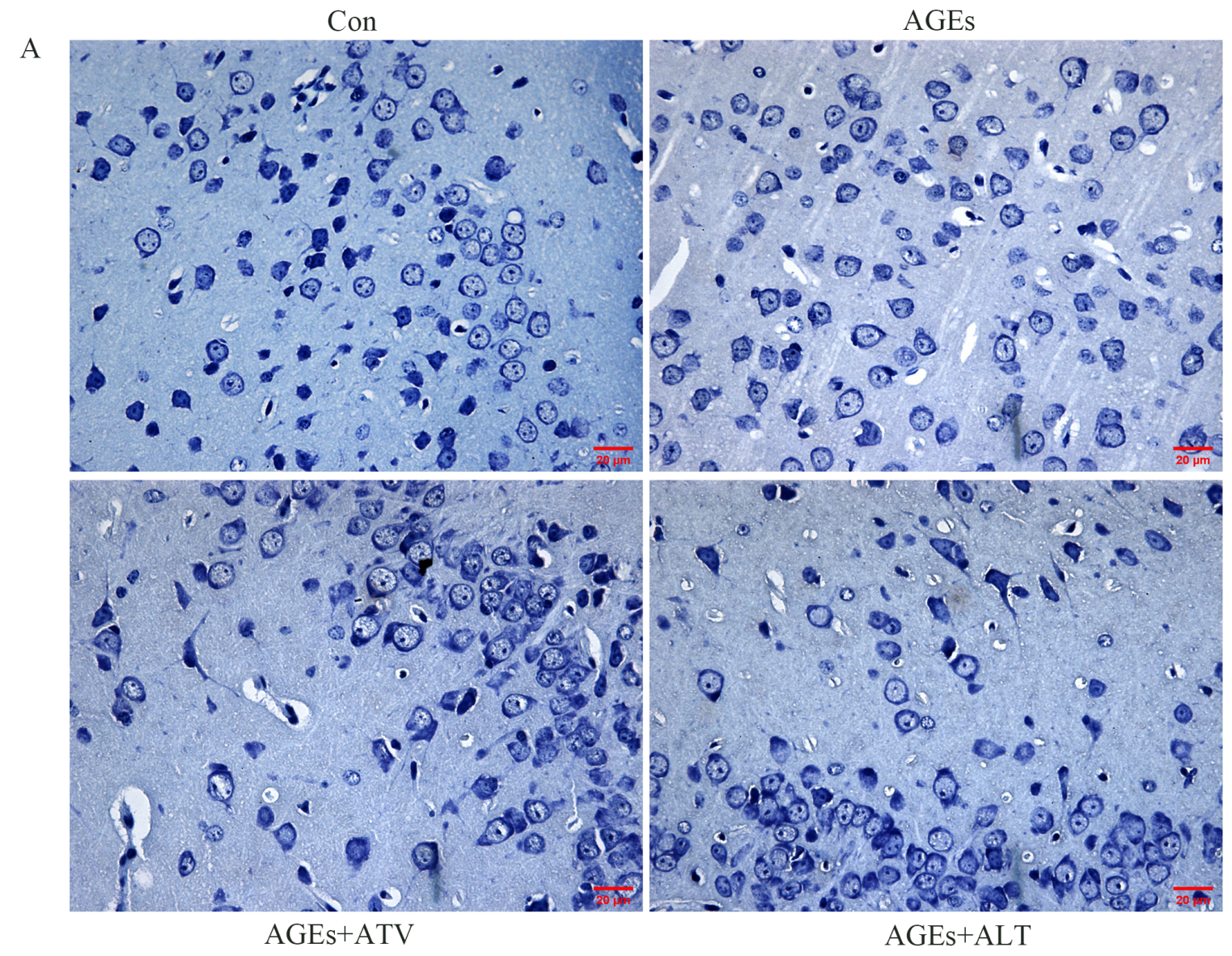

B

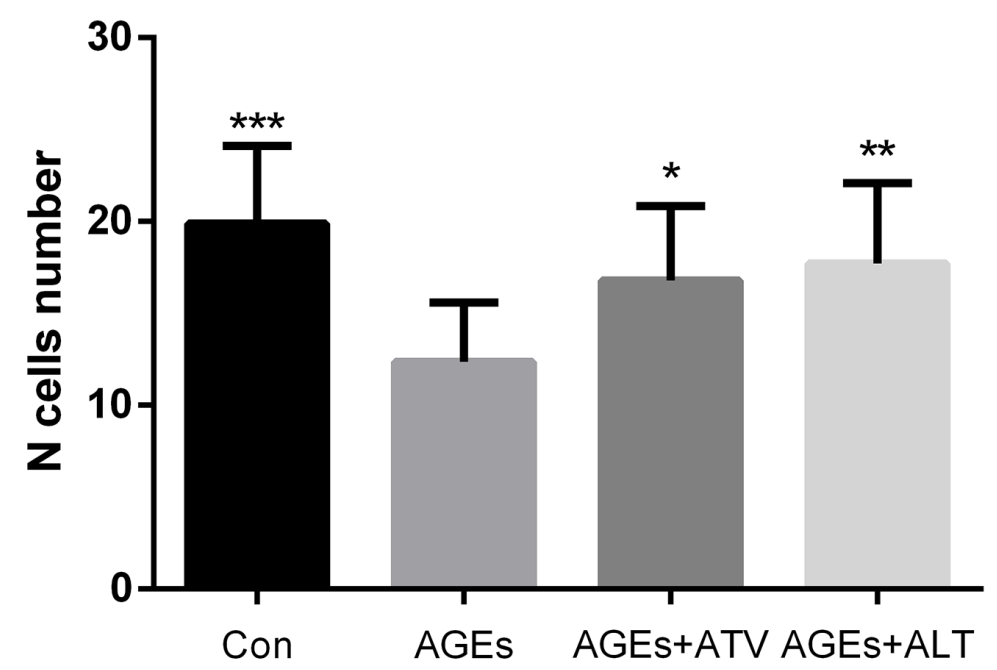

Figure 2

The functional state of neurons. Brain sections with Nissl's Staining ( $A, \times 400$ magnification). Number of Nissl bodies in cerebral cortex(B). GraphPad values are presented as mean $\pm \mathrm{SEM}$. $* \mathrm{P}<0.05$ versus the AGEs group, $*^{*} \mathrm{P}<0.01$ versus the AGEs group and $*^{\star *} \mathrm{P}<0.001$ versus the AGEs group. 


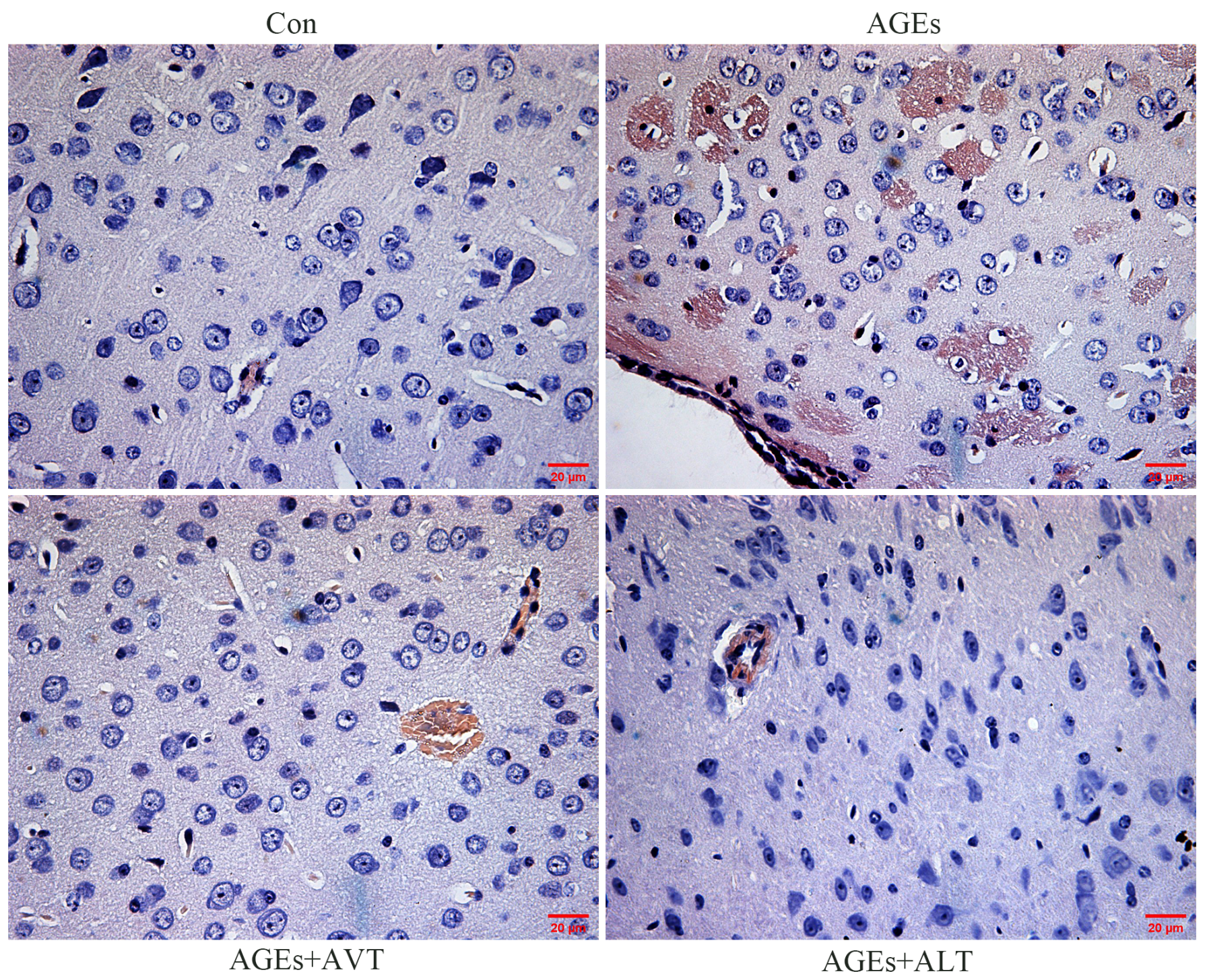

Figure 3

$A \beta$ plaque formation in cerebral cortex. Brain sections with Congo Red histochemical stain $(\times 200$ magnification). 

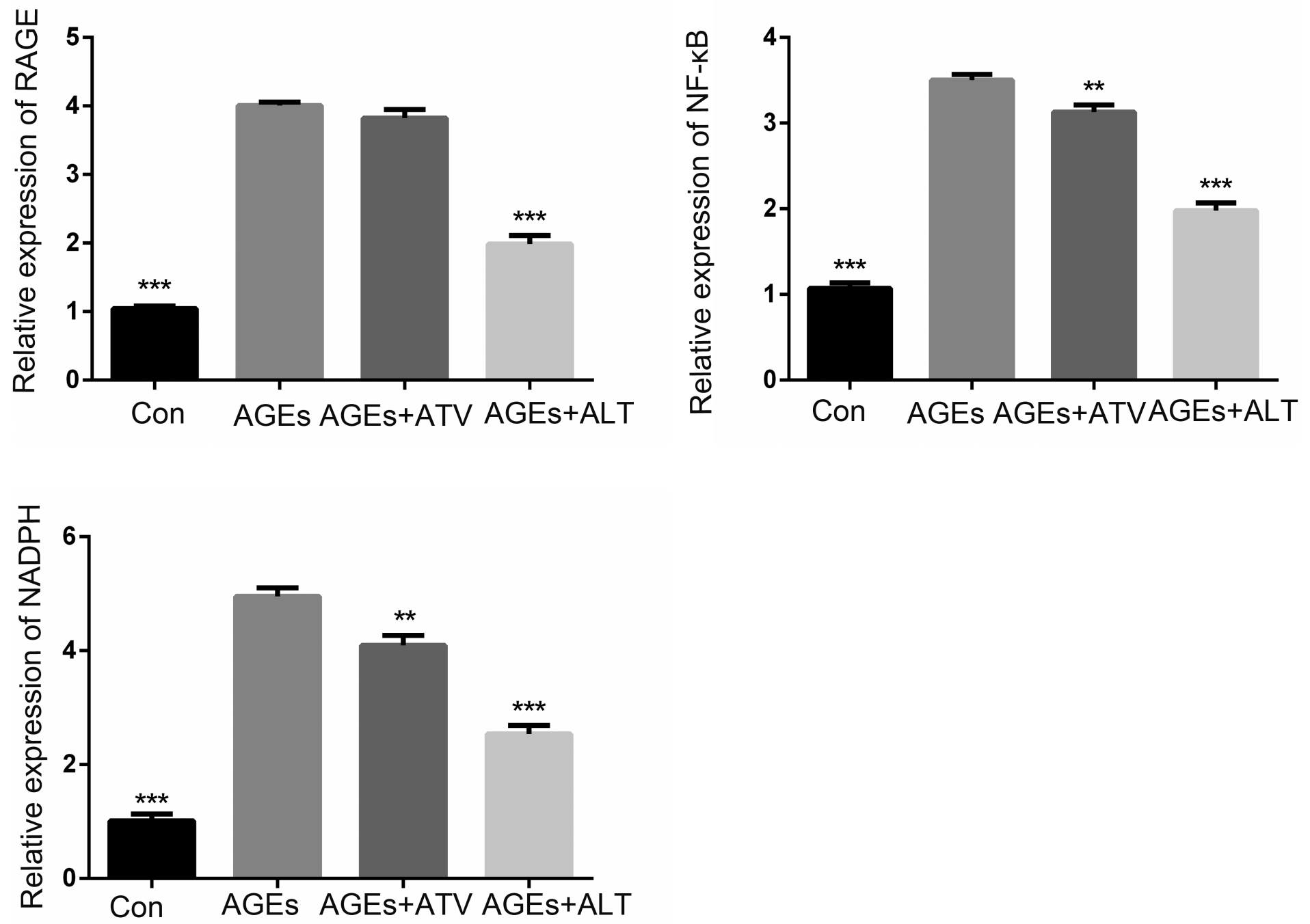

Figure 4

Expression of RAGE, NADPH oxidase p47phox and NF-kB p65 in cerebral cortex by qRT-PCR. GraphPad values are presented as mean $\pm S E M . * P<0.05$ versus the AGEs group, $* * P<0.01$ versus the AGEs group and $* * * P<0.001$ versus the AGEs group. 


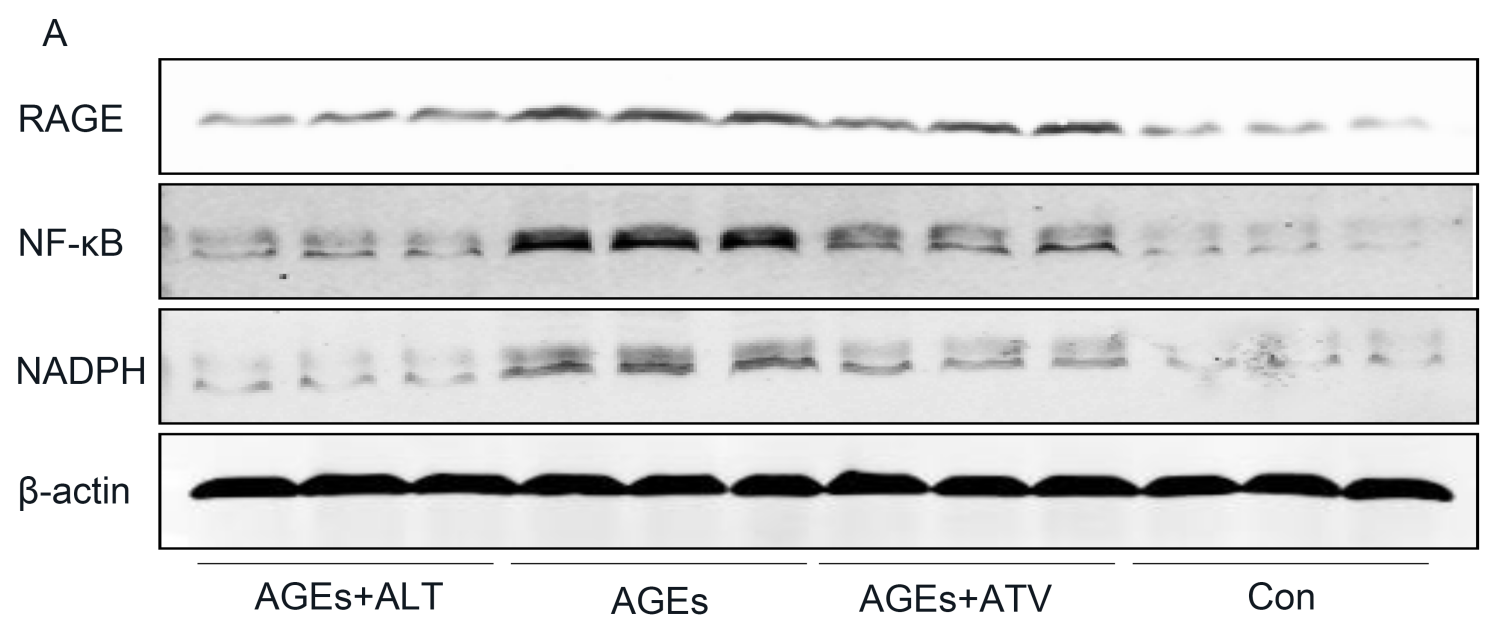

B
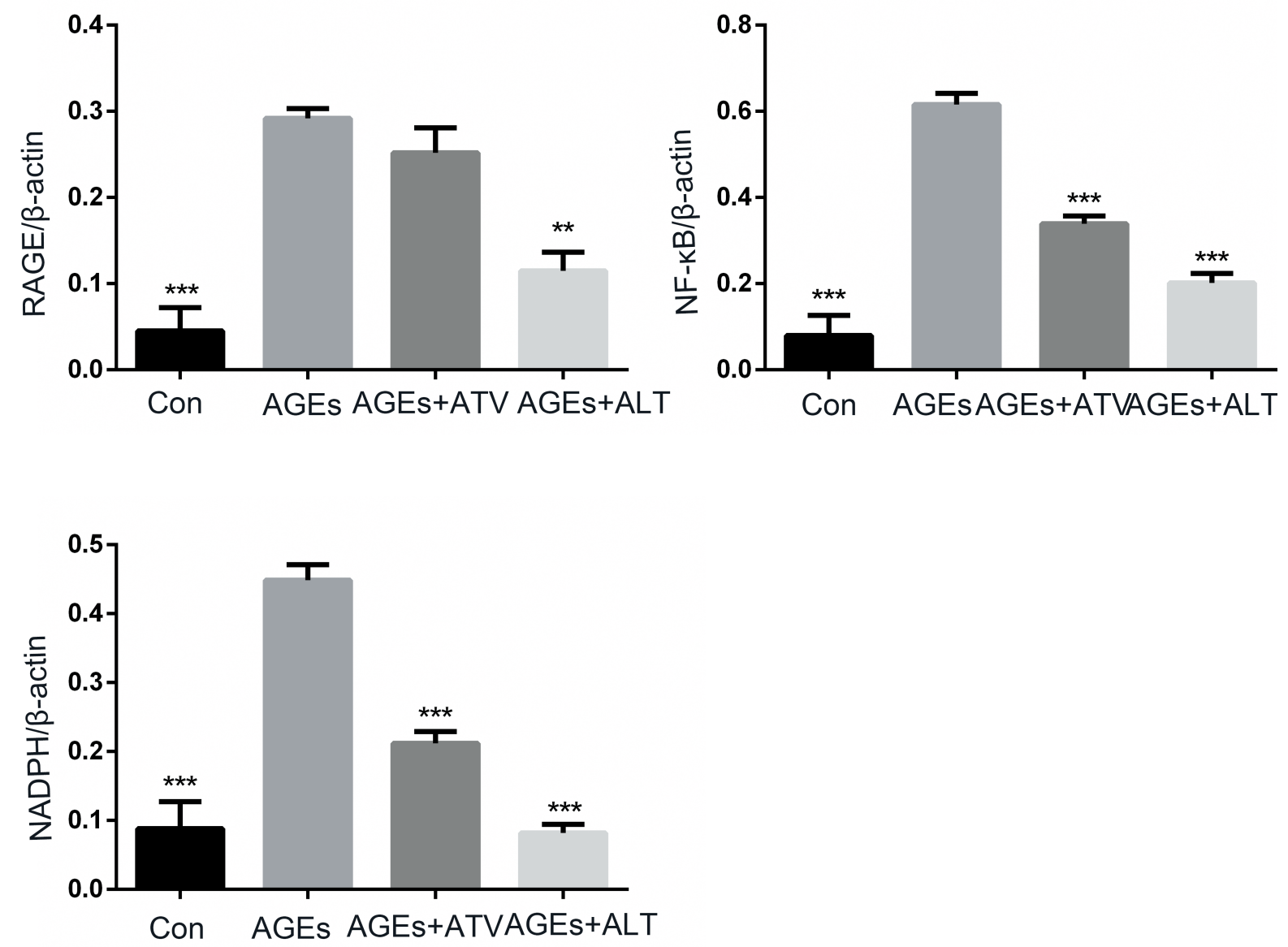

Figure 5

Western blot was used to reflect the protein levels of RAGE, NADPH oxidase p47phox and NF-KB p65 in cerebral cortex (A). GraphPad values are presented as mean $\pm \mathrm{SEM} . * \mathrm{P}<0.05$ versus the AGEs group, $*{ }^{*} \mathrm{P}<0.01$ versus the AGEs group and $*{ }^{* *} \mathrm{P}<0.001$ versus the AGEs group. 